\title{
Corrigendum: Long lifespan in worms with long telomeric DNA
}

K S Joeng, E J Song, K-J Lee \& J Lee

Nat. Genet. 36, 607-611 (2004).

The authors acknowledge that the work was also supported in part by a grant from the KRF.

\footnotetext{
Corrigendum: Mutations in the desmosomal protein plakophilin-2 are common in arrhythmogenic right ventricular cardiomyopathy

B Gerull, A Heuser, T Wichter, M Paul, C T Basson, D A McDermott, B B Lerman, S M Markowitz, P T Ellinor, C A MacRae, S Peters, K S Grossman, B Michely, S Sasse-Klaassen, M Birchmeier, R Dietz, G Breithardt, E Schulze-Bahr \& L Thierfelder Nat. Genet. 36, 1162-1164 (2004).

Jörg Drenckhahn should have been included as the thirteenth author of this paper. His affiliation is Max-Delbrück Center for Molecular Medicine, D-13092 Berlin-Buch, Germany.

\section{Corrigendum: Widespread occurrence of alternative splicing at NAGNAG acceptors contributes to proteome plasticity}

M Hiller, K Huse, K Szafranski, N Jahn, J Hampe, S Schreiber, R Backofen \& M Platzer

Nat. Genet. 36, 1255-1257 (2004).

There is a seeming inconsistency between Supplementary Tables 1 and 4 online. Several human-mouse conserved NAGNAG acceptors (Supplementary Table 4 online) do not appear among all human NAGNAG acceptors in the CDS (Supplementary Table 1 online), because we used a more rigid filtering procedure for Supplementary Table 1: only NAGNAG acceptors in unambiguous CDS RefSeq annotations were listed. In addition, Supplementary Table 4 online also shows human-mouse conserved NAGNAG acceptors in the UTR and in CDS with errors or ambiguous characters. A revised version of Supplementary Table 4 is available upon request from the authors.
} 